This is a pre-print of an article published in Graefes Arch Clin Exp Ophthalmol (2017) 255:91-97. The final authenticated version is available online at: https://doi.org/10.1007/s00417-016-3520-x

\title{
Arginase activity, urea concentration and hydroxyproline synthesis are reduced in keratoconus keratocytes
}

Tanja Stachon ${ }^{1}$, Krasimir Kolev ${ }^{2}$, Zsuzsa Flaskó ${ }^{3}$, Berthold Seitz ${ }^{1}$, Achim

Langenbucher ${ }^{4}$, Nóra Szentmáry ${ }^{1,5}$

${ }^{1}$ Department of Ophthalmology, Saarland University Medical Center, Homburg/Saar, Germany

${ }^{2}$ Department of Medical Biochemistry, Semmelweis University, Budapest, Hungary

${ }^{3}$ Kenézy Hospital, Department of Ophthalmology, Debrecen, Hungary

${ }^{4}$ Experimental Ophthalmology, Saarland University, Homburg/Saar, Germany

${ }^{5}$ Department of Ophthalmology, Semmelweis University, Budapest, Hungary

Running head: Arginase activity, urea and hydroxyproline in keratoconus keratocytes

Correspondence: Tanja Stachon, Department of Ophthalmology, Saarland University Medical Center, Kirrberger Str. 100, D-66424 Homburg/Saar, Germany

Telephone number: 0049-6841-1622387

Fax number: 0049-6841-1622400

E-Mail: tanja.stachon@uks.eu 
This is a pre-print of an article published in Graefes Arch Clin Exp Ophthalmol (2017) 255:91-97. The final authenticated version is available online at: https://doi.org/10.1007/s00417-016-3520-x

\section{ABSTRACT}

Purpose: Keratoconus $(\mathrm{KC})$ is a disease characterized by thinning and deformation of the cornea, but its etiology remains unknown. Seventy percent of the corneal stroma consists of collagen, which is composed of three intertwined polypeptide chains with glycine-hydroxyproline-proline repeats along their sequence. Arginase is a cytoplasmatic enzyme and catalyzes the conversion of arginine to urea and ornithine, which serves as a precursor for the endogenous synthesis of proline and hydroxyproline. The purpose of this study was to analyze arginase activity, as well as collagen and urea formation in normal and KC-keratocytes and to determine the impact of urea on keratocyte viability and proliferation, in vitro.

Methods: Primary human keratocytes were isolated by digestion in collagenase (1.0 $\mathrm{mg} / \mathrm{ml}$ ) from surgically removed corneas of 8 keratoconus patients and 8 normal human corneal buttons, and cultured in DMEM/Ham's F12 medium supplemented with $10 \%$ fetal calf serum. Arginase activity and urea concentration were measured in cell-lysates, hydroxyproline concentration in supernatant of cultured keratocytes using colorimetric assay. Cell viability and cell proliferation of cultured keratocytes were assessed after treatment with urea at concentrations up to $10 \mathrm{mM}$ for $24 \mathrm{~h}$ using assays for metabolic activity and DNA replication.

Results: Arginase activity and urea concentration in KC-keratocytes decreased by about $50 \%$ compared to normal keratocytes $(\mathrm{p}=0.003$ and $\mathrm{p}=0.008)$. Hydroxyproline synthesized by cultured KC-keratocytes was also approximately $50 \%$ less compared to normal keratocytes $(\mathrm{p}=0.02)$ and this difference decreased following treatment with 5.0 or $10.0 \mathrm{mM}$ urea $(\mathrm{p}=0.02 ; 0.03)$, without any change in cell viability $(\mathrm{p}>0.09)$. However, the urea treatment increased modestly (by $20 \%$ ) the proliferation rate of KC-keratocytes $(p=0.04 ; 0.04 ; 0.04)$, without any effect on normal cultured 
This is a pre-print of an article published in Graefes Arch Clin Exp Ophthalmol (2017) 255:91-97. The final authenticated version is available online at: https://doi.org/10.1007/s00417-016-3520-x

keratocytes $(\mathrm{p}>0.09)$.

Conclusions: We identified suppressed arginase activity in the metabolic program of cultured keratoconus keratocytes. The level of urea, as one product of the enzyme arginase was also decreased. This results in impaired collagen synthesis, evidenced in the culture by reduced hydroxyproline synthesis. In addition, our data showed that the other product of the arginase reaction, urea supports the proliferation of KC-keratocytes, without changes in their viability. The metabolic reprogramming of keratoconus keratocytes and its impact on development of a clinically detectable keratoconus disease has to be further analyzed.

Key words: Urea, keratoconus, keratocytes, arginase, hydroxyproline concentration, viability, proliferation 
This is a pre-print of an article published in Graefes Arch Clin Exp Ophthalmol (2017) 255:91-97. The final authenticated version is available online at: https://doi.org/10.1007/s00417-016-3520-x

\section{INTRODUCTION}

Keratoconus $(\mathrm{KC})$ is a disease characterized by thinning and deformation of the cornea. Patients develop first symptoms usually in early teens to early twenties with an incidence of about $1 / 2,000$ [1]. Keratoconus starts usually during puberty with progression until the third decade of life [2]. The etiology of keratoconus remains unknown, but some researchers suggest involvement of inflammatory enzymes beside genetical components [3-5]. Different proteases like matrix-metalloproteinase-1 (MMP-1) [6], MMP-9 and MMP-13 [7] may be involved in keratoconus disease. Thinning of a keratoconus cornea occurs with degradation of the extracellular matrix. Seventy percent of the corneal stroma consists of collagen, predominantly of type I collagen (80-90\%) and of collagen $\mathrm{V}[8,9]$. The fibril-forming collagens are composed of triple helical subunits, the stability of which is based on the repeating glycine-X-Y amino acid triplets in the structure of the intertwined polypeptide chains (38\% of position $\mathrm{X}$ in these repeats is occupied by hydroxyproline and $28 \%$ of position Y by proline) [10] . Hydroxyproline is formed by a posttranslational hydroxylation of proline in protocollagen and thus its quantity depends on the rate of collagen synthesis.

Arginase is an essential enzyme in tissue regeneration, cell proliferation and anti-inflammation [11]. Arginase, as a cytoplasmic enzyme catalyzes the conversion of arginine to urea and ornithine. Ornithine will be further converted by ornithine aminotransferase and pyrroline-5-carboxylate reductase to proline, which is incorporated in protocollagen, the precursor of collagen and some of these proline residues are further transformed to hydroxyproline (Figure 1). Therefore, arginase may play an essential role in collagen production of the cells, also in keratoconus patients. 
This is a pre-print of an article published in Graefes Arch Clin Exp Ophthalmol (2017) 255:91-97. The final authenticated version is available online at: https://doi.org/10.1007/s00417-016-3520-x

Interestingly, in a previous study, we detected decreased urea concentration in aqueous humour of keratoconus patients, compared to controls [12]. In addition, Jäger et al. have shown that urea concentration in tear film of dry eye subjects is decreased compared to healthy controls [13]. Keratoconus is often associated with dry-eye syndrome (DES), which results in symptoms of discomfort, tear film instability caused by increased osmolarity of the tear film [14].

These findings raise the hypothesis that changes related to urea metabolism may be part of the keratoconus disease phenotype. In view of the known disturbances of collagen turnover in keratoconus patients, the current study was undertaken in attempt to address the possibility that impaired arginase-dependent supply of proline could result in impaired collagen synthesis and thus contribute to the pathomechanism of keratoconus disease.

Therefore, in the current study, our purpose was to analyze arginase activity, as well as urea concentration and hydroxyproline synthesis of normal and keratoconus keratocytes and to define the impact of urea on keratocyte viability and proliferation. 
This is a pre-print of an article published in Graefes Arch Clin Exp Ophthalmol (2017) 255:91-97. The final authenticated version is available online at: https://doi.org/10.1007/s00417-016-3520-x

MATERIALS AND METHODS

\section{Materials}

Dulbecco's Modified Eagle Medium: (Nutrient Mixture F-12 (DMEM/F12)); fetal calf serum (10\%); P/S (1\% of $10000 \mathrm{u}$ penicillin/ $10 \mathrm{mg}$ streptomycin per $\mathrm{ml}) ; 0.05 \%$ trypsin/ $0.02 \%$ ethylenediaminetetra-acetic acid (EDTA), PBS, CelLytic ${ }^{\mathrm{TM}}$, Urea assay kit and Arginase activity assay kit were purchased from Sigma-Aldrich ${ }^{\circledR} \mathrm{GmbH}$ (Deisenheim, Germany), alamarBlue ${ }^{\circledR}$ from Invitrogen (Karlsruhe, Germany). Collagenase A, Dispase II and BrdU Proliferation-ELISA were obtained from Roche Diagnostics (Mannheim, Germany). Colorimetric assay for Hydroxyproline was purchased from Chondrex. Inc. (Redmond, USA).

\section{Isolation of primary human corneal keratocytes}

Eight human normal corneas were obtained from the Saarland University Medical Center Eye Bank and 8 corneas from keratoconus patients from elective penetrating keratoplasties. To isolate keratocytes, the human corneoscleral buttons were aseptically rinsed in phosphate-buffered saline (PBS) before removal of the endothelium including Descemet's membrane by sterile surgical disposable scalpel. A central corneal button with epithelium was cut using a $8.0 \mathrm{~mm}$ hand-held trephine and thereafter incubated in culture medium containing $2.4 \mathrm{U} / \mathrm{ml}$ Dispase II for 4 hours at $37^{\circ} \mathrm{C}$. Thereafter, the corneal button was washed with PBS for several times and the already loose corneal epithelium was removed with surgical disposable scalpel. The remaining corneal stroma was incubated in culture medium with $1.0 \mathrm{mg} / \mathrm{ml}$ collagenase A for $8-10$ hours at $37^{\circ} \mathrm{C}$. The digested tissue and cells were pipetted three times and centrifuged at $800 \mathrm{~g}$ for 7 minutes and finally resuspended in $1.0 \mathrm{ml}$ culture medium, which consisted of basic medium (DMEM/F12) supplemented with 
This is a pre-print of an article published in Graefes Arch Clin Exp Ophthalmol (2017) 255:91-97. The final authenticated version is available online at: https://doi.org/10.1007/s00417-016-3520-x

$10 \%$ FCS and $1 \% \mathrm{P} / \mathrm{S}$. The cell suspension was seeded in 6 -well plates and the medium was changed 24 hours after seeding. Medium was changed every 2 to 3 days until keratocytes reached confluence. The cells were subcultured in $25 \mathrm{~cm}^{2}$ culture flasks after 5 to 10 days following dispersal with $0.05 \%$ trypsin/ $0.02 \%$ ethylenediaminetetra-acetic acid (EDTA) for 3 to 5 minutes and the passage 4 to 8 of cells was used for experiments. Normal keratocyte cell cultures were used as control cells.

\section{Arginase activity measurement}

After that the cultured cells reached confluence, lysate of $3 \times 10^{6}$ normal $(n=8)$ or $3 \times$ $10^{6} \mathrm{KC}$ keratocytes $(\mathrm{n}=8)$ was prepared using $10 \mathrm{mM}$ Tris/HCL buffer ( $\left.\mathrm{pH} 7.4\right)$ containing $0.4 \%(\mathrm{w} / \mathrm{v})$ Triton $^{\mathrm{TM}} \mathrm{X}-100$ with additional 3 freeze and thaw cycles. Following centrifugation, the supernatant was used for the assay. The test was performed according to the manufacturer's protocol. Shortly, arginase from samples catalyzes the conversion of arginin to urea and ornithine. The produced urea specifically reacts with the substrate to generate a colored product, proportional to the arginase activity. The absorbance was measured at $550 \mathrm{~nm}$ using an absorbance microplate reader (TECAN infinite 50, TECAN Deutschland GmbH, Crailsheim, Germany). The arginase activity of each sample was calculated using the absorbance of a standard solution.

\section{Protein measurement}

After taking the supernatant for hydroxyproline determination, the total protein concentration of each well was measured to calculate the amount of hydroxyproline per mg protein, as follows. Following detachment of the cells with $150 \mu 1$ CelLytic $^{\mathrm{TM}}$, 
This is a pre-print of an article published in Graefes Arch Clin Exp Ophthalmol (2017) 255:91-97. The final authenticated version is available online at: https://doi.org/10.1007/s00417-016-3520-x

protein quantity was determined according to the method of Bradford, which is based on the formation of a complex between the dye, Brilliant blue $\mathrm{G}$ and proteins in solution. The absorbance was measured at $595 \mathrm{~nm}$ and the concentrations were quantified using bovine serum albumin (BSA) as standard.

\section{Urea concentration measurement in keratocytes}

Lysate of $5 \times 10^{6}$ normal $(n=5)$ and $\mathrm{KC}$ keratocytes $(\mathrm{KC})(\mathrm{n}=5)$ was prepared using 10 $\mathrm{mM}$ Tris/HCL buffer ( $\mathrm{pH} 7.4)$ containing $0.4 \%(\mathrm{w} / \mathrm{v})$ Triton ${ }^{\mathrm{TM}} \mathrm{X}-100$ with additional 3 freeze and thaw cycles. After centrifugation, the supernatant was used for the assay. The test was performed according to the manufacturer's protocol: Urea concentration was determined by a coupled enzyme reaction, which results in a colorimetric product, proportional to the urea concentration. The optical density was measured at $550 \mathrm{~nm}$ using an absorbance microplate reader (TECAN infinite 50). The urea concentration of each sample was calculated using the optical density of a standard solution.

\section{Urea treatment of keratocyte cell cultures before hydroxyproline measurement}

Keratocytes were seeded in a 24-multiwell plate at a density of $8 \times 10^{3} \mathrm{cells} / \mathrm{cm}^{2}$ in culture medium of $1 \mathrm{ml}$ per well. After a growth period of $48 \mathrm{~h}$ the culture medium was changed to culture medium containing urea concentrations of $0,5.0$ and $10.0 \mathrm{mM}$ for $24 \mathrm{~h}$ at $37^{\circ} \mathrm{C}$ for 24 hours. The supernatant was stored at $-80^{\circ} \mathrm{C}$ until hydroxyproline measurement.

\section{Hydroxyproline measurement}

Hydroxyproline measurement in the supernatant of urea treated keratocytes was performed by a colorimetric assay kit with linear detection ranges of $6 \mu \mathrm{g} / \mathrm{ml}-400$ 
This is a pre-print of an article published in Graefes Arch Clin Exp Ophthalmol (2017) 255:91-97. The final authenticated version is available online at: https://doi.org/10.1007/s00417-016-3520-x

$\mu \mathrm{g} / \mathrm{ml}$. The measured concentrations below the lower threshold of this range were considered as zero. The measurements were performed following the manufacturer's protocol. First, supernatant of keratocytes was hydrolyzed with $10 \mathrm{~N} \mathrm{HCL}$ at $120^{\circ} \mathrm{C}$ for 24 hours. Then, sample solutions were transferred to microcentrifuge tubes and were spun at 10,000 rpm for 3 minutes. Thereafter, the supernatant of the solution was pipetted into a 96 well-plate and was treated with chloramine-T and Ehrlich reagent (dimethylaminobenzaldehyde). Additional microplate wells were filled with known dilutions of hydroxyproline and were used to create a calibration curve. The optical density of each well was measured with an absorbance microplate reader at $550 \mathrm{~nm}$ (TECAN infinite 50). Hydroxyproline content in samples was then determined using regression analysis. In each well, the concentration of hydroxyproline was normalized to the cell protein concentration of the respective well.

\section{Keratocyte viability using different urea concentrations}

Cell viability was evaluated using the alamarBue ${ }^{\circledR}$ assay as follows: human keratocytes were seeded in 24-well cell culture plates at a density of $7.5 \times 10^{3}$ cells $/ \mathrm{cm}^{2}$. After a growth period of $24 \mathrm{~h}$, the culture medium was changed to culture medium containing urea concentrations of $0,0.5,1.0,5.0$ and $10.0 \mathrm{mM}$ at $37^{\circ} \mathrm{C}$ for 24 h. Subsequently alamarBlue ${ }^{\circledR}$ solution was diluted with culture medium for a final concentration of $10 \%$ and $500 \mu \mathrm{l}$ of this solution was added to each well. As a negative control, alamarBlue ${ }^{\circledR}$ solution was added to a well without cells. Absorbance was measured at $550 \mathrm{~nm}$ using a 96-well microplate reader (Tecan infinite F50).

\section{Keratocyte proliferation using different urea concentrations}

To detect the impact of urea on the proliferation rate of the keratocytes, the 
This is a pre-print of an article published in Graefes Arch Clin Exp Ophthalmol (2017) 255:91-97. The final authenticated version is available online at: https://doi.org/10.1007/s00417-016-3520-x

$-10-$

proliferation was determined with the proliferation ELISA-BrdU kit, by the measurement of BrdU(5-bromo-2'deoxyuridine) incorporation in the newly synthesized cellular DNA. Keratocytes were seeded in a 96-multiwell plate at a density of $6 \times 10^{3}$ cells $/ \mathrm{cm}^{2}$ in culture medium of $100 \mu 1$ per well. After a growth period of $24 \mathrm{~h}$, the culture medium was changed to culture medium containing urea at $0,0.5,1.0,5.0$ and $10.0 \mathrm{mM}$ at $37^{\circ} \mathrm{C}$ for 24 hours. The test was performed according to the manufacturer's protocol. Briefly, BrdU was added to the keratocytes and incubated at $37^{\circ} \mathrm{C}$ for $4 \mathrm{~h}$. After removing the culture medium, the cells were fixed with FixDenat, provided with the test kit, followed by the incubation with anti-BrdU-POD (peroxidase), which binds the incorporated DNA. After adding the substrate solution, the immune complex was detected using an absorbance microplate reader (Tecan Infinite 50).

\section{Statistical Analysis}

For statistical analysis the GraphPad Prism 2.01 was used. Data are represented as mean \pm standard deviation (SD). Statistical analysis was performed using Wilcoxon-Mann-Whitney Test. P values below 0.05 were considered statistically significant. 
This is a pre-print of an article published in Graefes Arch Clin Exp Ophthalmol (2017) 255:91-97. The final authenticated version is available online at: https://doi.org/10.1007/s00417-016-3520-x

$-11-$

\section{RESULTS}

Arginase activity in KC-keratocytes was significantly decreased compared to normal keratocytes ( $\mathrm{p}=0.003$ ), as shown in Figure 2. In line with this finding the concentration of the arginase reaction product, urea was also significantly lower in cultured KC-keratocytes $(5.36 \mathrm{ng} / \mu \mathrm{l})$ than in normal keratocytes $(8.89 \mathrm{ng} / \mu \mathrm{l} ; \mathrm{p}=0.008)$ $(p=0.008)$ (Figure 3). The significantly lower hydroxyproline concentration in the KC-keratocyte cultures indicated impaired collagen synthesis compared to normal keratocytes (Figure 4), although treatment with 5.0 or $10.0 \mathrm{mM}$ urea did not change significantly the viability of KC-keratocytes compared to controls ( $p>0.09$ ) (data not shown). The difference in the hydroxyproline content of normal and $\mathrm{KC}$-keratocytes decreased after 24-h urea exposure, but remained significant $(p=0.02$ and 0.03 )

(Figure 4). The treatment of normal keratocytes with different urea concentrations did not change cell proliferation significantly $(\mathrm{p}>0.09)$. However, proliferation of KC-keratocytes was significantly $(\mathrm{p}<0.05)$ increased by exposure to $0.5,1.0$ or 10.0 $\mathrm{mM}$ urea, compared to keratoconus keratocyte cultures without urea treatment (Figure 5). 
This is a pre-print of an article published in Graefes Arch Clin Exp Ophthalmol (2017) 255:91-97. The final authenticated version is available online at: https://doi.org/10.1007/s00417-016-3520-x

$-12-$

\section{DISCUSSION}

Keratoconus is a severe disease of the cornea, resulting in thinning and conical shape

[1]. There is an association between keratoconus and collagen disorders like

Ehlers-Danlos syndrome and mitral valve prolapse [2]. However, the exact causes of keratoconus remain unknown.

In patients with keratoconus, the tissue develops biomechanical instability, which results in conical shape. The production of collagen by corneal keratocytes is a major factor in biomechanical stability and shape of the cornea [15]. Impairment of collagen synthesis may be a possible reason for the development of keratoconus.

Recent years literature on the role of arginase in the immune system increased enormously, as it has a crucial role in various aspects of inflammation such as inflammation triggered immune dysfunction, tumor immune escape, immunosuppression and immunopathology of infectious disease [16]. Arginase is a manganese metalloenzyme, which plays a major role in collagen synthesis. Arginase is responsible for the conversion of L-arginine to L-ornithine and urea. L-ornithine is further converted to proline. Proline is partially hydroxylated into hydroxyproline that stabilizes the triple helical subunits of collagen [17]. Adequate amount and activity of the enzyme arginase is essential for proline supply in keratocytes for collagen synthesis (Figure 1).

In our current study, we found impaired arginase activity in human $\mathrm{KC}$ keratocytes, compared to normal keratocytes. The most conspicuous finding of our study is that arginase activity, urea concentration and hydroxyproline synthesis are reduced in keratoconus keratocytes. Decreased arginase activity could be proven through two independent methods in our study. First, we directly measured decreased arginase activity in KC keratocytes compared to normal keratocytes. Second, as an indirect 
This is a pre-print of an article published in Graefes Arch Clin Exp Ophthalmol (2017) 255:91-97. The final authenticated version is available online at: https://doi.org/10.1007/s00417-016-3520-x

$-13-$

measurement, the level of urea, as one product of the enzyme arginase was also

decreased. Decreased urea concentration in aqueous humour of $\mathrm{KC}$ patients could also be verified in our previous work, compared to controls [12]. These previous in vivo measurement data further support results of our present in vitro experiments.

With decreased arginase activity in KC keratocytes we have also found decreased hydroxyproline synthesis in KC keratocytes, compared to normal controls. It is to note, that the hydroxyproline concentration in keratocyte cultures is decreased, despite the supplemented L-arginine and L-proline in the culture medium. This supports the conclusion that the exogenous proline is insufficient for cells with active collagen synthesis that require excessive amounts of this amino acid. The arginase activity of keratocytes may be the limiting step in the synthesis of collagen.

All of the above data (decreased arginase activity, urea concentration and hydroxyproline synthesis) suggest an arginase-dependent impairment of collagen synthesis in keratoconus keratocytes. Whether arginase activity is impaired or the level of arginase expression is reduced through inherited or acquired factors remains to be further clarified.

Another novel aspect of our work was the effect of urea on the viability, proliferation and hydroxyproline synthesis of normal and KC keratocytes. We found that urea treatment stimulated the proliferation of KC-keratocytes, but not of normal keratocytes without any changes in the viability of either cell type. Interestingly, Moeslinger et al. also found a proliferation promoting effect of urea on macrophages [18].

Keratocytes show a downregulated MYC-gene (MYC, proto-oncogene protein), which is involved in cell cycle regulation [19]. MYC is a transcription factor that can bind the DNA and activate several regulation processes. The activation of MYC can 
This is a pre-print of an article published in Graefes Arch Clin Exp Ophthalmol (2017) 255:91-97. The final authenticated version is available online at: https://doi.org/10.1007/s00417-016-3520-x

$-14-$

promote cell growth and proliferation [20]. Urea may be involved in cell signaling to

activate the MYC-gene for cell proliferation [19] or increasing urea concentrations

slow down arginase activity and through the accumulation of arginine enhance cell

proliferation [11]. These pathways have to be further analyzed in keratoconus patients.

A further reason for the decreased amount of hydroxyproline may be the reduced concentration of L-arginine through the competing nitric oxide (NO) pathway (Figure

1). If the collagen production is impaired, NO pathway may also show altered

processes. In addition, it is suggested, that the development of keratoconus is also

associated with increased levels of reactive oxygen species - these may also be

involved in alterations of keratoconus corneas [21]. Therefore, further experiments

concerning NO production via nitric oxide synthase have to be performed using

keratoconus corneas.

In summary, arginase activity, urea concentration and hydroxyproline synthesis are reduced in keratoconus keratocytes. Urea treatment of keratoconus keratocytes does not change viability, but stimulate proliferation of these cells. The metabolic reprogramming of keratoconus keratocytes and its impact on development of a clinically detectable keratoconus disease has to be further analyzed. 
This is a pre-print of an article published in Graefes Arch Clin Exp Ophthalmol (2017) 255:91-97. The final authenticated version is available online at: https://doi.org/10.1007/s00417-016-3520-x

\section{ACKNOWLEDGEMENTS}

We thank the Alexander von Humboldt Foundation for supporting the work of Dr. N.

Szentmáry at the Department of Ophthalmology of Saarland University Medical Center in Homburg/Saar, Germany. Dr K. Kolev received support from the Hungarian Scientific Research Fund (grant OTKA 112612). 
This is a pre-print of an article published in Graefes Arch Clin Exp Ophthalmol (2017) 255:91-97. The final authenticated version is available online at: https://doi.org/10.1007/s00417-016-3520-x

$-16-$

\section{References}

1. Krachmer JH, Feder RS, Belin MW (1984) Keratoconus and related noninflammatory corneal thinning disorders. Surv Ophthalmol 28:293-322.

2. Rabinowitz YS (1998) Keratoconus. Surv Ophthalmol 42:297-319.

3. Lema I, Duran JA (2005) Inflammatory molecules in the tears of patients with keratoconus. Ophthalmology 112:654-659.

4. Abu-Amero KK, Al-Muammar AM, Kondkar AA (2014) Genetics of keratoconus: where do we stand? J Ophthalmol 2014:641708.

5. Jun AS, Cope L, Speck C, Feng X, Lee S, Meng H, Hamad A, Chakravarti S (2011) Subnormal cytokine profile in the tear fluid of keratoconus patients. PLoS One 6:e16437.

6. Collier SA (2001) Is the corneal degradation in keratoconus caused by matrix-metalloproteinases? Clin Experiment Ophthalmol 29:340-344.

7. Balasubramanian SA, Mohan S, Pye DC, Willcox MDP (2012) Proteases, proteolysis and inflammatory molecules in the tears of people with keratoconus. Acta Ophthalmol 90:e303-9.

8. Gloor M, Fluhr J, Lehmann L, Gehring W, Thieroff-Ekerdt R (2002) Do urea/ammonium lactate combinations achieve better skin protection and hydration than either component alone? Skin Pharmacol Appl Skin Physiol $15: 35-43$.

9. Chen S, Mienaltowski MJ, Birk DE (2015) Regulation of corneal stroma extracellular matrix assembly. Exp Eye Res 133:69-80.

10. Jenkins CL, Bretscher LE, Guzei IA, Raines RT (2003) Effect of 3-hydroxyproline residues on collagen stability. J Am Chem Soc $125: 6422-6427$. 
This is a pre-print of an article published in Graefes Arch Clin Exp Ophthalmol (2017) 255:91-97. The final authenticated version is available online at: https://doi.org/10.1007/s00417-016-3520-x

$-17-$

11. Munder M (2009) Arginase: an emerging key player in the mammalian immune system. Br J Pharmacol 158:638-651.

12. Stachon, T; Stachon, A, Hartmann, U; Seitz, B; Langenbucher, A; Szentmáry N (2015) Abstract-Band DOG 2015. Der Ophthalmol 112:1-180.

13. Jager K, Kielstein H, Dunse M, Nass N, Paulsen F, Sel S (2013) Enzymes of urea synthesis are expressed at the ocular surface, and decreased urea in the tear fluid is associated with dry-eye syndrome. Graefes Arch Clin Exp Ophthalmol 251:1995-2002.

14. Zemova E, Eppig T, Seitz B, Toropygin S, Arnold S, Langenbucher A, Graber S, Szentmary N (2014) Interaction between topographic/tomographic parameters and dry eye disease in keratoconus patients. Curr Eye Res 39:1-8.

15. Vellara HR, Patel D V. (2015) Biomechanical properties of the keratoconic cornea: A review. Clin Exp Optom 98:31-38.

16. Bronte V, Zanovello P (2005) Regulation of immune responses by L-arginine metabolism. Nat Rev Immunol 5:641-654.

17. Caldwell RB, Toque HA, Narayanan SP, Caldwell RW (2015) Arginase: an old enzyme with new tricks. Trends Pharmacol Sci 36:395-405.

18. Moeslinger T, Friedl R, Volf I, Brunner M, Baran H, Koller E, Spieckermann PG (1999) Urea induces macrophage proliferation by inhibition of inducible nitric oxide synthesis. Kidney Int 56:581-588.

19. Mace M, Galiacy SD, Erraud A, Mejia JE, Etchevers H, Allouche M, Desjardins L, Calvas P, Malecaze F (2011) Comparative transcriptome and network biology analyses demonstrate antiproliferative and hyperapoptotic phenotypes in human keratoconus corneas. Invest Ophthalmol Vis Sci $52: 6181-6191$. 
This is a pre-print of an article published in Graefes Arch Clin Exp Ophthalmol (2017) 255:91-97. The final authenticated version is available online at: https://doi.org/10.1007/s00417-016-3520-x

20. Stine, ZE; Walton, ZE; Altmann, BJ; Hsieh, AL; Dang V (2015) MYC, Metabolism and Cancer. 8:1699-1712.

21. Cejkova J, Cejka C (2015) The role of oxidative stress in corneal diseases and injuries. Histol Histopathol 30:893-900. 
This is a pre-print of an article published in Graefes Arch Clin Exp Ophthalmol (2017) 255:91-97. The final authenticated version is available online at: https://doi.org/10.1007/s00417-016-3520-x

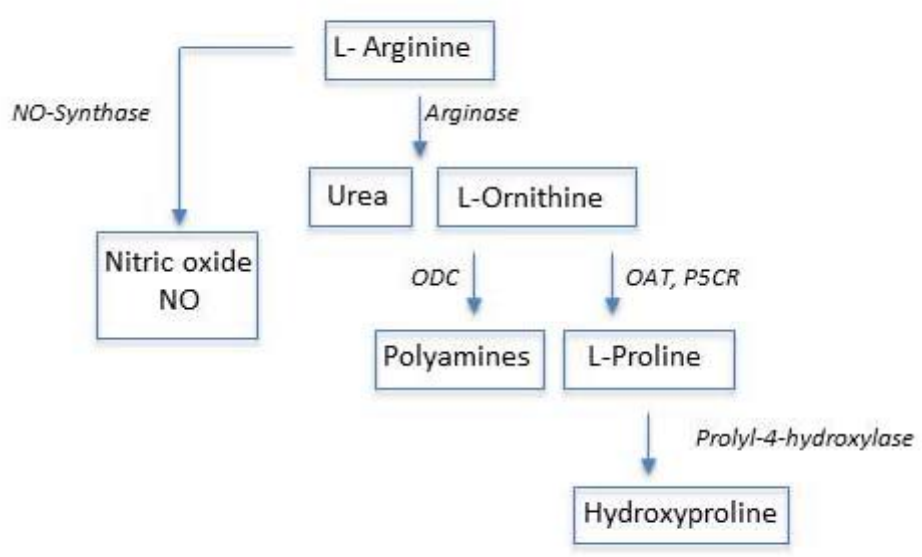

Figure 1. Arginase, as a cytoplasmic enzyme catalyzes the conversion of L-arginine to L-ornithine and urea. Ornithine will be further transformed by the sequential action of ornithine aminotransferase (OAT) and pyrroline-5-carboxylate reductase (P5CR) to proline. Following the translation of protocollagen, some of its proline residues are hydroxylated to hydroxyproline by prolyl-4-hydroxylase. Ornithine is also converted by the enzyme ornithine decarboxylase (ODC) to polyamines. The competing enzyme for the substrate L-arginine is nitric-oxide synthase (NO-Synthase), which catalyzes the reaction to nitric oxide. 
This is a pre-print of an article published in Graefes Arch Clin Exp Ophthalmol (2017) 255:91-97. The final authenticated version is available online at: https://doi.org/10.1007/s00417-016-3520-x

\section{Arginase activity in keratocytes}

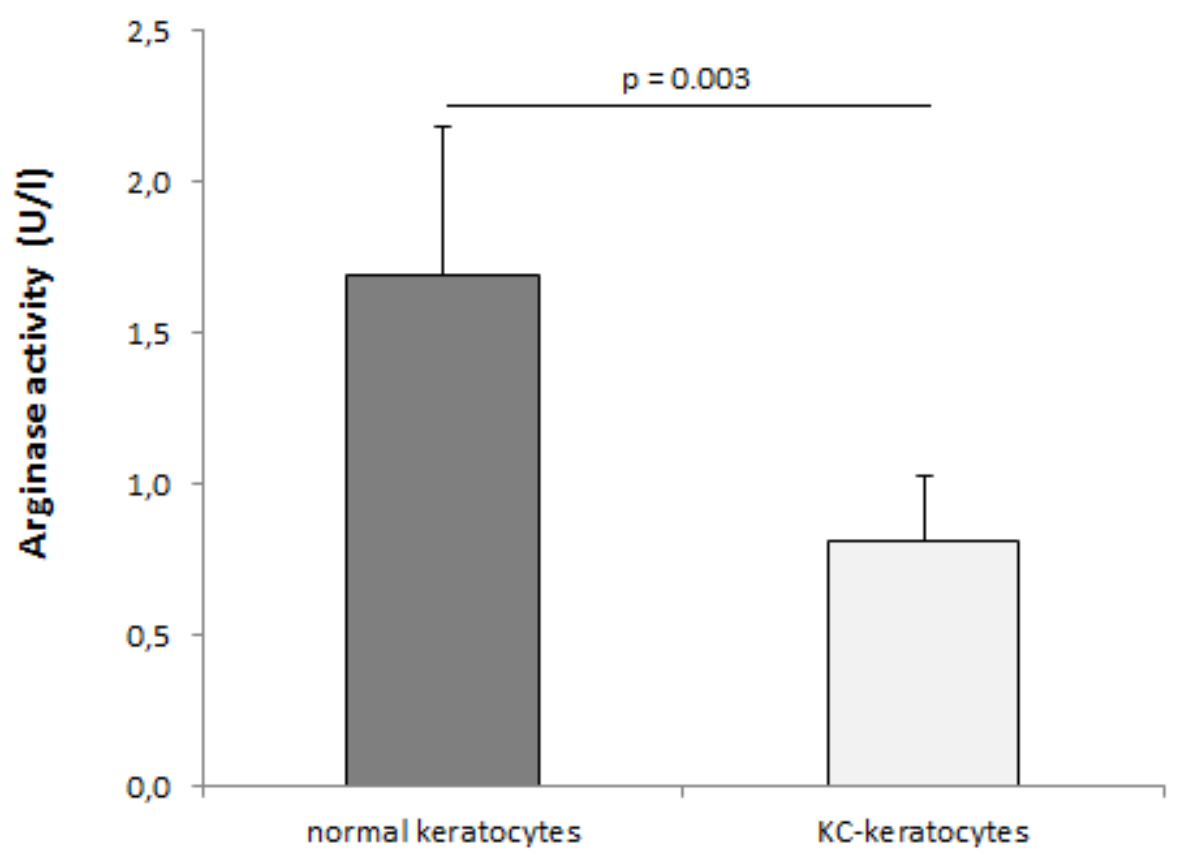

Figure 2. Arginase activity in normal and keratoconus (KC) keratocytes. Significant difference is indicated. 
This is a pre-print of an article published in Graefes Arch Clin Exp Ophthalmol (2017) 255:91-97. The final authenticated version is available online at: https://doi.org/10.1007/s00417-016-3520-x

\section{Urea concentration in keratocytes}

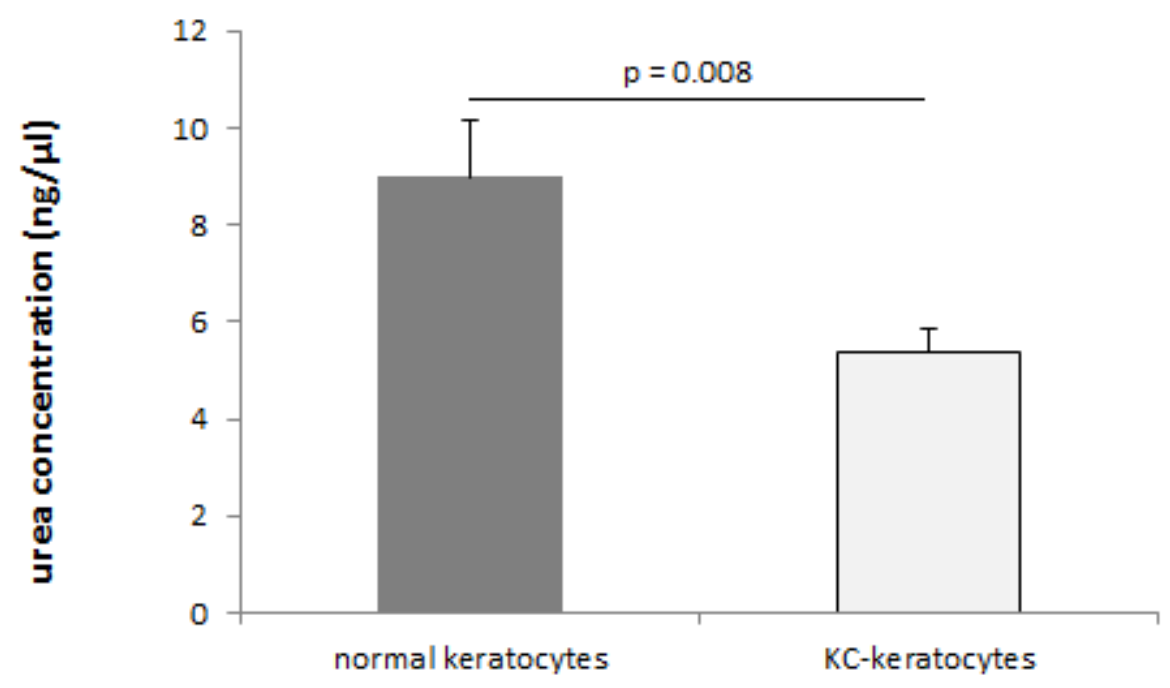

Figure 3. Urea concentration in normal and keratoconus (KC) keratocytes. Significant difference is indicated. 
This is a pre-print of an article published in Graefes Arch Clin Exp Ophthalmol (2017) 255:91-97. The final authenticated version is available online at: https://doi.org/10.1007/s00417-016-3520-x

\section{Hydroxyproline synthesis of keratocytes}

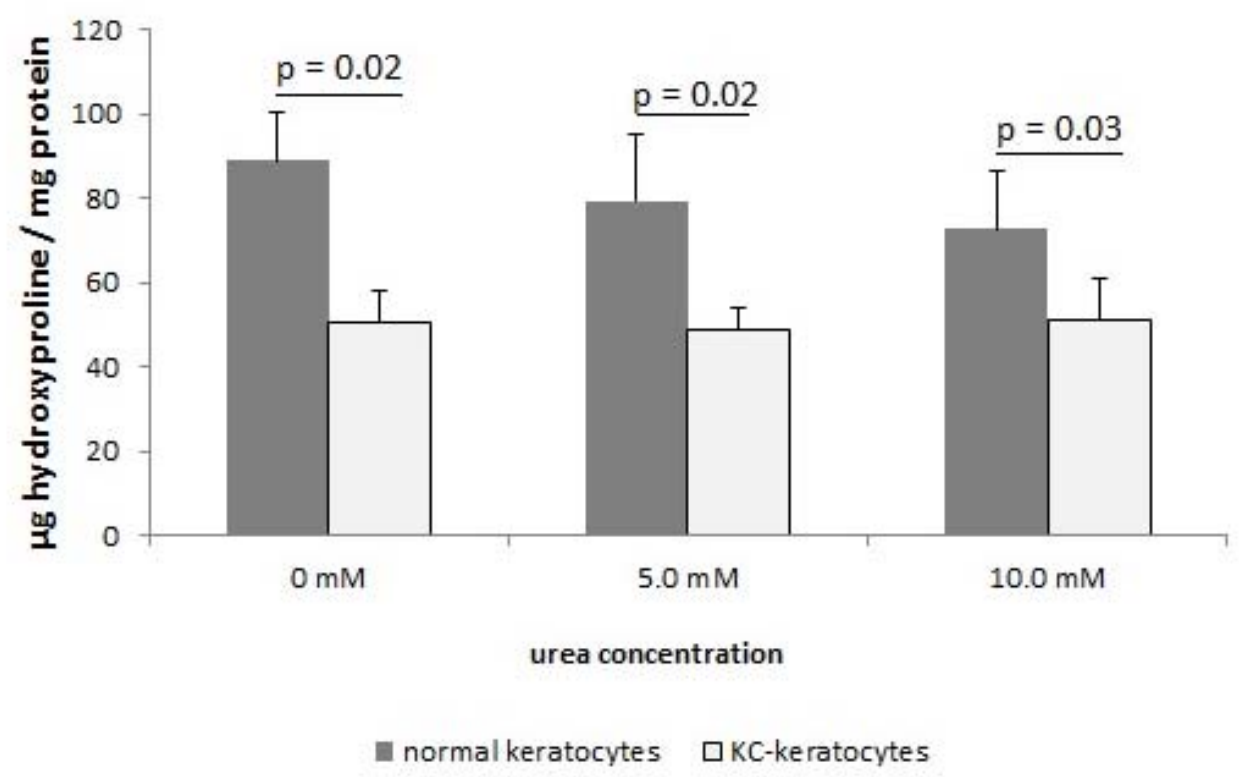

Figure 4. Hydroxyproline synthesis in cultures of normal and keratoconus (KC) keratocytes 24 hours after urea treatment. Hydroxyproline synthesis of keratocytes was significantly less in keratoconus cultures compared to normal keratocytes adding $0,5.0$ or $10.0 \mathrm{mM}$ concentrations of urea to the cultures $(p=0.02,0.02$ and 0.03$)$. 
This is a pre-print of an article published in Graefes Arch Clin Exp Ophthalmol (2017) 255:91-97. The final authenticated version is available online at: https://doi.org/10.1007/s00417-016-3520-x

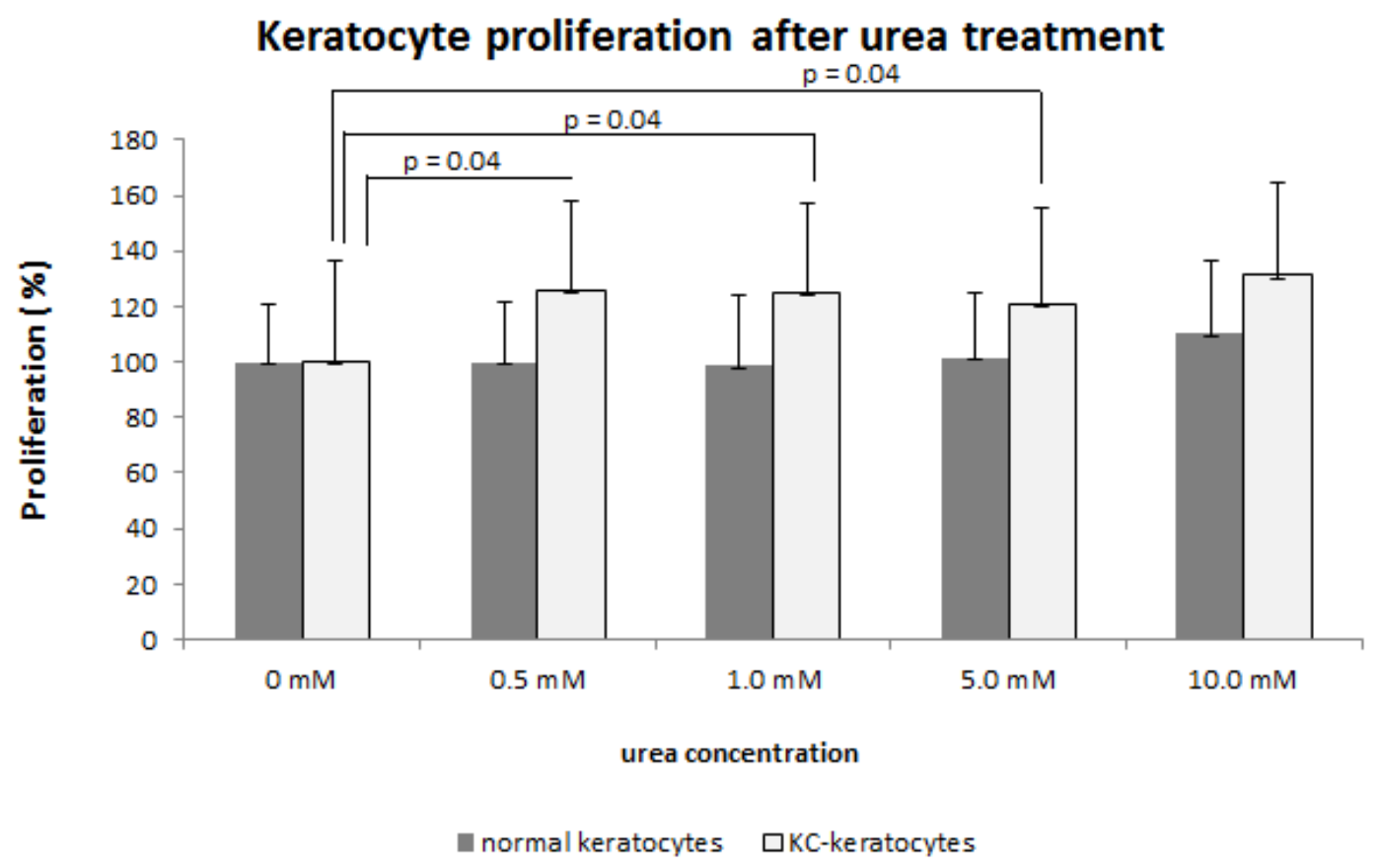

Figure 5. Proliferation rate of normal and keratoconus (KC) keratocytes.

Incorporation of BrdU(5-bromo-2'deoxyuridine) in the newly synthesized cellular

DNA was measured after 24-h exposure to urea at the indicated concentrations in the culture medium. The absorbance of untreated keratocytes was set to $100 \%$, significant differences are indicated.

The treatment of normal keratocytes with different urea concentrations did not change keratocyte proliferation significantly compared to control cells $(\mathrm{p}>0.09)$. However, proliferation of $\mathrm{KC}$ keratocytes was significantly increased using $0.5,1.0$ or $10.0 \mathrm{mM}$ concentrations of urea $(p=0.04 ; 0.04 ; 0.04)$, compared to KC keratocyte cultures without urea treatment. 